\title{
Effect of concentrated growth factors on beagle periodontal ligament stem cells in vitro
}

\author{
BOHAN YU and ZUOLIN WANG \\ Center of Implant Dentistry, Hospital of Stomatology, Tongji University, Shanghai 200092, P.R. China
}

Received April 2, 2013; Accepted October 14, 2013

DOI: $10.3892 / \mathrm{mmr} .2013 .1756$

\begin{abstract}
Identifying a reliable and effective cytokine or growth factor group has been the focus of stem cell osteogenic induction studies. Concentrated growth factors (CGFs) as the novel generation of platelet concentrate products, appear to exhibit a superior clinical and biotechnological application potential, however, there are few studies that have demonstrated this effect. This study investigated the proliferation and differentiation of periodontal ligament stem cells (PDLSCs) co-cultured with CGFs. The rate of proliferation was analyzed by cell counting and an MTT assay. Mineralization nodule counts, alkaline phosphatase activity detection, qPCR, western blot analysis and immunohistochemistry were used to analyze mineralization effects. The results showed that CGF significantly promoted the proliferation of PDLSCs, and exhibited a dose-dependent effect on the activation and differentiation of the stem cells. The application of CGF on PDLSC proliferation and osteoinduction may offer numerous clinical and biotechnological application strategies.
\end{abstract}

\section{Introduction}

The increased lifespan of human beings has led to the development of novel therapeutic strategies aiming to preserve aged tissue, which range from the replacement of lost or injured tissues to regeneration of the damage tissue (1). Regenerating alveolar bone as an alternative therapy for periodontal regeneration has been increasingly used in dentistry. A deficient alveolar ridge not only fails to provide sufficient support and retention for teeth or dentures, but also hinders dental implant placement (2). The finding of stem cells was a medical breakthrough and led to the novel field of medicine entitled regenerative medicine (3). Postnatal stem cells have

Correspondence to: Dr Zuolin Wang, Center of Implant Dentistry, Hospital of Stomatology, Tongji University, No. 399 Middle Yanchang Road, Shanghai 200092, P.R. China

E-mail: zuolin@tongji.edu.cn

Key words: concentrated growth factors, periodontal ligament stem cells, co-culture, proliferation, osteo-induction, dose-dependent effect been isolated from various tissues, including dental tissue (4). Seo et al suggested that the human periodontal ligament (PDL) contains a population of postnatal multipotent stem cells (5). Stem cells require specific microenvironments for survival (6), thus, in theory periodontal ligament stem cells (PDLSCs) are the most direct and reliable source for periodontal tissue regeneration. Although PDLSCs are easy to separate and purify, the number of cells required for bone regeneration is unachievable in a short period of time. After several passages, it is difficult to induce the differentiation of cells that are prone to aging and changes in phenotype $(7,8)$. Thus, an effective way to rapidly amplify PDLSCs is a current focus of numerous studies.

Growth factors stimulate the differentiation of mesenchymal cells into osteoblasts and accelerate the osteogenesis of these cells. The growth factors that are known to be associated with bone formation are bone morphogenetic protein (BMP), transforming growth factor (TGF), platelet-derived growth factor (PDGF), insulin-like growth factor (IGF), and epidermal growth factor (EGF) (9-11). When these growth factors are used alone, there are often certain shortcomings. For example, when using BMP-2 alone, it has a short halflife and is susceptible to proteolytic degradation. When the concentration is too high, it may lead to tissue edema, an increased inflammatory response, and prevention of novel bone formation (12-14). In addition, the human body is a complex biological environment; thus, stem cell osteogenic differentiation is not regulated by one growth factor alone. Therefore, it is essential to identify an autologous source and effective cytokine or growth factor group in stem cell osteogenic induction studies. Platelet-rich plasma (PRP) (15), a platelet concentrate product, contains numerous growth factors. Previously, it was demonstrated that the PRP may induce proliferation, but inhibits the differentiation of PSLSCs (16). The application of PRP has also been controversial as the addition of dissimilar thrombin and anticoagulant during preparation may pose risks of immune rejection and transmission of infectious diseases. The platelet-rich fibrin (PRF), the second generation of platelet concentrate products (17), exhibits the same properties as PRP with the advantages of an osteogenic ability, a simple preparation process, and lack of added biological agents, as it is produced from autologus blood. Concentrated growth factors (CGF) were developed by Sacco in 2006. It is produced by a centrifuge device (Medifuge Silfradent srl, Italy), similar to the production of PRF (18). The different centrifugation speed 
permits the isolation of fibrin matrix that is markedly larger, denser and richer in growth factors as compared to PRF. In theory, CGFs appear to exhibit superior clinical and biotechnological application potential (19); however, there are few studies supporting this.

The aim of this study was to analyze the in vitro biological effects of CGFs on the proliferation and differentiation of canine PDLSCs, and to investigate whether this effect occurs in a dose-dependent manner.

\section{Materials and methods}

Isolation of beagle PDLSCs and preliminary identification. One healthy beagle dog (18 months old, $14.5 \mathrm{~kg}$, male) was supplied by the Laboratory Center of the Second Military Medical University of the Chinese PLA (No. SCXK-Shanghai 2012-0003). The entire experimental procedure was in accordance with the Regulations of the Administration of Affairs Concerning Experimental Animals formulated by the Ministry of Science and Technology of China. Beagle PDLSCs were isolated and cultured as described previously $(4,20,21)$. Briefly, periodontal ligament tissues were scraped from the intermediate $1 / 3$ of the root. Collagenase type I $(3 \mathrm{mg} / \mathrm{ml}$; Sigma-Aldrich, St. Louis, MO, USA) and dispase II (4 mg/ml; Roche, Basel, Switzerland) were added at a 1:1 mixture and digested for $1 \mathrm{~h}$ at $37^{\circ} \mathrm{C}$. The mixture was passed through a $70 \mu \mathrm{m}$ cell sieve (BD Biosciences Franklin Lakes, NJ, USA), using $\alpha$-minimal essential medium (MEM) containing $15 \%$ fetal bovine serum (FBS) to adjust the cell density to $1 \times 10^{4} / \mathrm{ml}$. The cells were then incubated in a $5 \% \mathrm{CO}_{2}$ atmosphere at $37^{\circ} \mathrm{C}$. Following expansion, the STRO- $1^{+}$cells (Biolegend, San Diego, CA, USA) were separated by flow cytometry. The cells were then inoculated on $\alpha$-MEM growth medium (Gibco-BRL, Carlsbad, CA, USA) containing 20\% FBS (Gibco), $2 \mathrm{mM}$ L-glutamine, $100 \mu \mathrm{M}$ L-ascorbate-2-phosphate, $1 \mathrm{mM}$ sodium pyruvate, $50 \mathrm{U} / \mathrm{ml}$ penicillin and $50 \mu \mathrm{g} / \mathrm{ml}$ streptomycin (21). The logarithmic growth phase cells underwent osteogenic, chondrogenic and adipogenic multi-induction experiments, and the induced cells were stained on day 21 with Alizarin Red S (Sigma-Aldrich), Alcian blue (Sigma-Aldrich) and Oil Red O (Sigma-Aldrich) accordingly.

CGF preparation. It was essential to use cultured PDLSCs and CGFs from the same donor. Under sterilized conditions, venous blood was drawn from the beagle forearm, divided into sterile Vacuette tubes (1 and $3 \mathrm{ml}$ samples in each tube, respectively) without anticoagulants and immediately placed in a centrifuge for centrifugation. The built-in program was: 30" acceleration, 2' $408 \times$ g, 4' 323 x g, 4' 408 x g, 3' 3,000 rpm, and 36" deceleration and stop. The fresh whole blood was divided into three layers (Fig. 1A). The intermediate filament protein gel layer was collected and pressed onto membranous film (MF200, Wisdom, Beijing, China; Fig. 1B).

Experimental groups. There were 6 groups used in the present study, the standard group (0 CGF), standard group + 1 CGFs (prepared with $1 \mathrm{ml}$ fresh whole blood), standard group +3 CGFs (prepared with $3 \mathrm{ml}$ whole blood), osteogensis induced fluid (0 CGFs), osteogensis induced fluid + 1 CGF and osteogensis induced fluid +3 CGFs. CGFs were added on the first
Table I. List of primers.

\begin{tabular}{ll}
\hline Gene & \multicolumn{1}{c}{ Sequences $\left(5^{\prime}-3^{\prime}\right)$} \\
\hline BSP & F: CGATTTCCAGTTCAGAGCAGTAGT \\
& R: CAGCGTCGGATTCATCTTCAT \\
Collagen I & F: TGGGGCAAGACAGTGATCG \\
& R: GGAGGGAGTTTACAGGAAGCAG \\
OCN & F: GCTGTGGCCGCACTCTGC \\
& R: AGAGTGGGGCTGGCCGCTC \\
GAPDH & F: AAGGTCGGAGTCAACGGATTT \\
& R: GGTTCACGCCCATCACAAA
\end{tabular}

BSP, bone sialoprotein 2; OCN, osteocalcin; GAPDH, glyceraldehyde 3-phosphate dehydrogenase.

day and experimental check points were at days 3 (D3), 7, 14 and 21 following inoculation.

The standard culture medium was composed of $\alpha$-MEM growth medium as described previously. The osteogenic medium (21) was composed of $\alpha$-MEM supplemented with $10 \%$ FBS, 2 mM L-glutamine, $100 \mu \mathrm{M}$ L-ascorbate-2phosphate, $10^{-7} \mathrm{M}$ dexamethasone, $1.8 \mathrm{mM}$ inorganic phosphate $\left(\mathrm{KH}_{2} \mathrm{PO}_{4}\right), 50 \mathrm{U} / \mathrm{ml}$ penicillin and $50 \mu \mathrm{g} / \mathrm{ml}$ streptomycin.

Cell growth and proliferation analysis. PDLSC proliferation increased following CGF treatment and was analyzed by cell counting and an MTT assay. The absorbance value of each well was measured at $490 \mathrm{~nm}$ by the microtiter analyzer (Multiskan FC; Thermo, Vantaa Finland). According to the measured OD values, with time as the $\mathrm{x}$-axis and absorbance value as the $y$-axis, the cell growth curve was plotted.

Osteoinduction assessment. The mineralized nodules were counted by Alizarin Red S (Sigma-Aldrich, St. Louis, MO, USA) staining and mineralized nodule formation was observed under the microscope (BX43; Olympus, Tokyo, Japan).

Alkaline phosphatase (ALP) activity detection was conducted using a commercialized reagent cartridge (?, USA) at each experimental check point and was analyzed in the microtiter analyzer at OD $405 \mathrm{~nm}$. The ALP activity was expressed as $\mathrm{nU} /$ cell and $\mathrm{mU} /$ plate.

qPCR was conducted using TRIzol reagent to extract RNA, and using a reverse transcription kit (Takara, Tokyo, Japan) to synthesize cDNA. Primers are listed in Table I. A two-step amplification response procedure was selected with specifications of $95^{\circ} \mathrm{C}$ for $120 \mathrm{sec}, 95^{\circ} \mathrm{C}$ for $15 \mathrm{sec}$ and $61^{\circ} \mathrm{C}$ for $40 \mathrm{sec}$ for 40 cycles.

For the western blot analysis, RIPA lysis buffer was added, homogenated and centrifuged and the protein supernatant was collected for sodium dodecyl sulphate-polyacrylamide gel electrophoresis. The proteins were transferred to nitrocellulose membranes, sealed and incubated with primary [BSP (Abcam, Cambridge, MA, USA), Col-I (Abcam) and OCN (Abcam)] and secondary antibodies (ZSGB-Bio, Beijing, China). The membranes were washed and immunoblot chemiluminescence detection reagents (enhanced chemiluminescence, ECL), were 


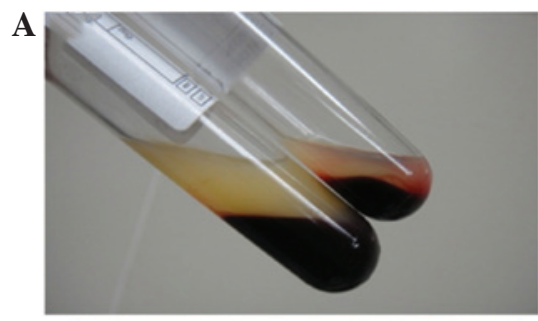

$\mathbf{C}$

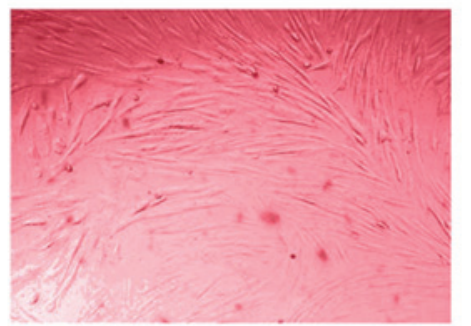

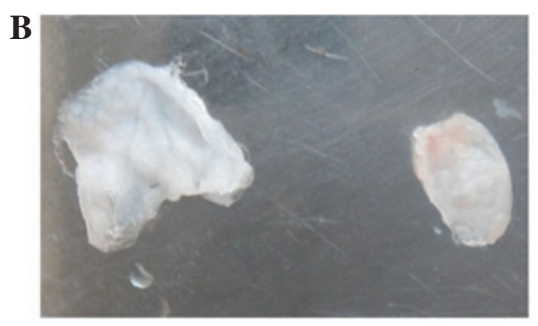

D

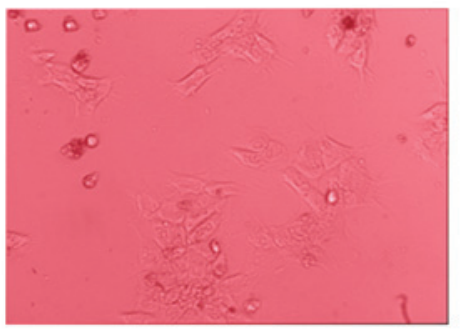

$\mathbf{E}$
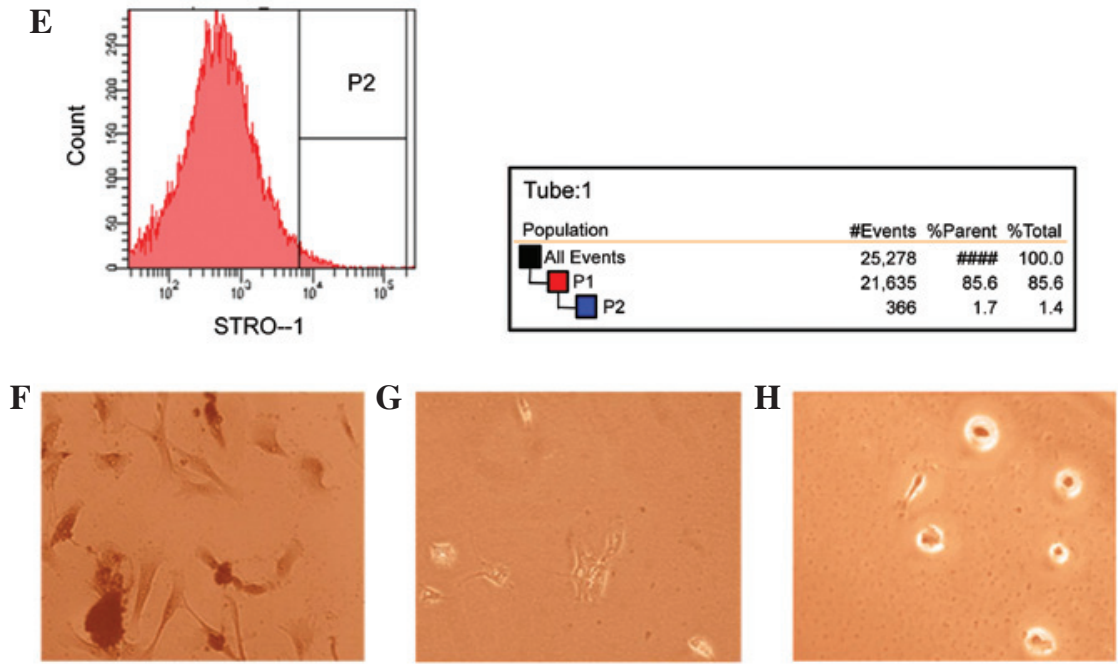

Figure 1. CGFs were prepared with (A) 1 and $3 \mathrm{ml}$ fresh whole blood and CGFs were pressed into (B) membranous film. (C) Primary PDLCs were obtained from beagle periodontal ligament; and (D) STRO-1 $1^{+}$PDLSCs were isolated by a flow cytometry. (E) The separation rate by flow cytometry was $1.7 \%$. PDLSCs were divided into osteogenic, chondrogenic and adipogenic induction groups and stained by (F) Alizarin Red S, (G) Alcian blue and (H) Oil Red O. The result showed a positive reaction. CGFs, concentrated growth factors; PDLCs, periodontal ligament stem cells.

used for exposure. Glyceraldehyde 3-phosphate dehydrogenase protein served as a system internal reference and stripe gray values were measured. The results were compared based on the differences in the expression of purpose/internal reference ratio.

For immunohistochemical detection, cell climbing film was produced, sealed and incubated with primary and secondary antibodies. DAB, chromogenic and hematoxylin staining was conducted, and the samples were sealed and observed under the microscope.

Statistical analysis. Cell count, ALP activity, mineralized nodule numbers and the positive expression rate of immunohistochemical staining were presented as the mean \pm SE and compared with the control group.

For the MTT assay, the survival capacity of the control group was set as $100 \%$. The experimental groups were calculated as percentages of the control group. $\mathrm{P}<0.05$ was considered to indicate a statistically significant difference.

Relative quantification of qPCR results were obtained using the comparative $\mathrm{Ct}$ method. According to the equation
Fold $=2^{-\Delta \Delta C t}$, the differences in the relative expression of the target gene of the experimental and control groups were calculated.

Western blot analysis results of three experiments were compared with the protein expression differences by scanning the striped gray value.

\section{Results}

Cell culture, activity and proliferation. Flow cytometry was used to screen PDLSCs (Fig. 1D) from PDLCs (Fig. 1C); the separation rate was $1.7 \%$ (Fig. 1E). To assess the differentiation capacity of PDLSCs, the separated PDLSCs were divided into osteogenic, chondrogenic and adipogenic induction groups. The cells were then stained by Alizarin Red S (Fig. 1F), Alcian blue (Fig. 1G) and Oil Red O (Fig. 1H). The results showed a positive reaction which indicated that the PDLSCs exhibited stem cell-specific differentiation ability.

Fig. 2A shows the proliferation effects of CGFs on PDLSCs. CGFs were identified to increase PDLSC proliferation in a 
A

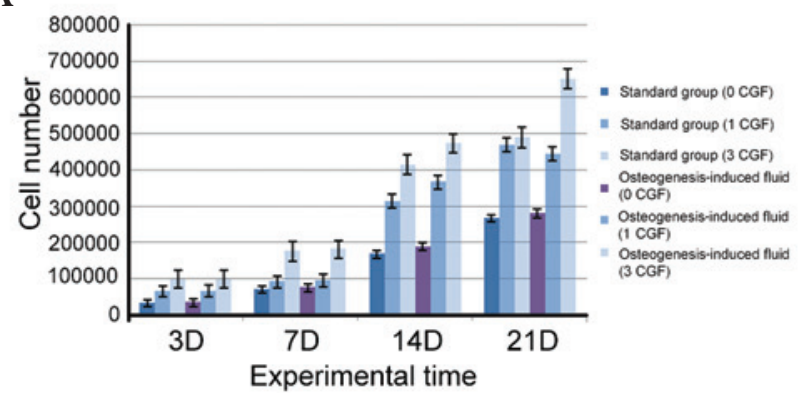

C
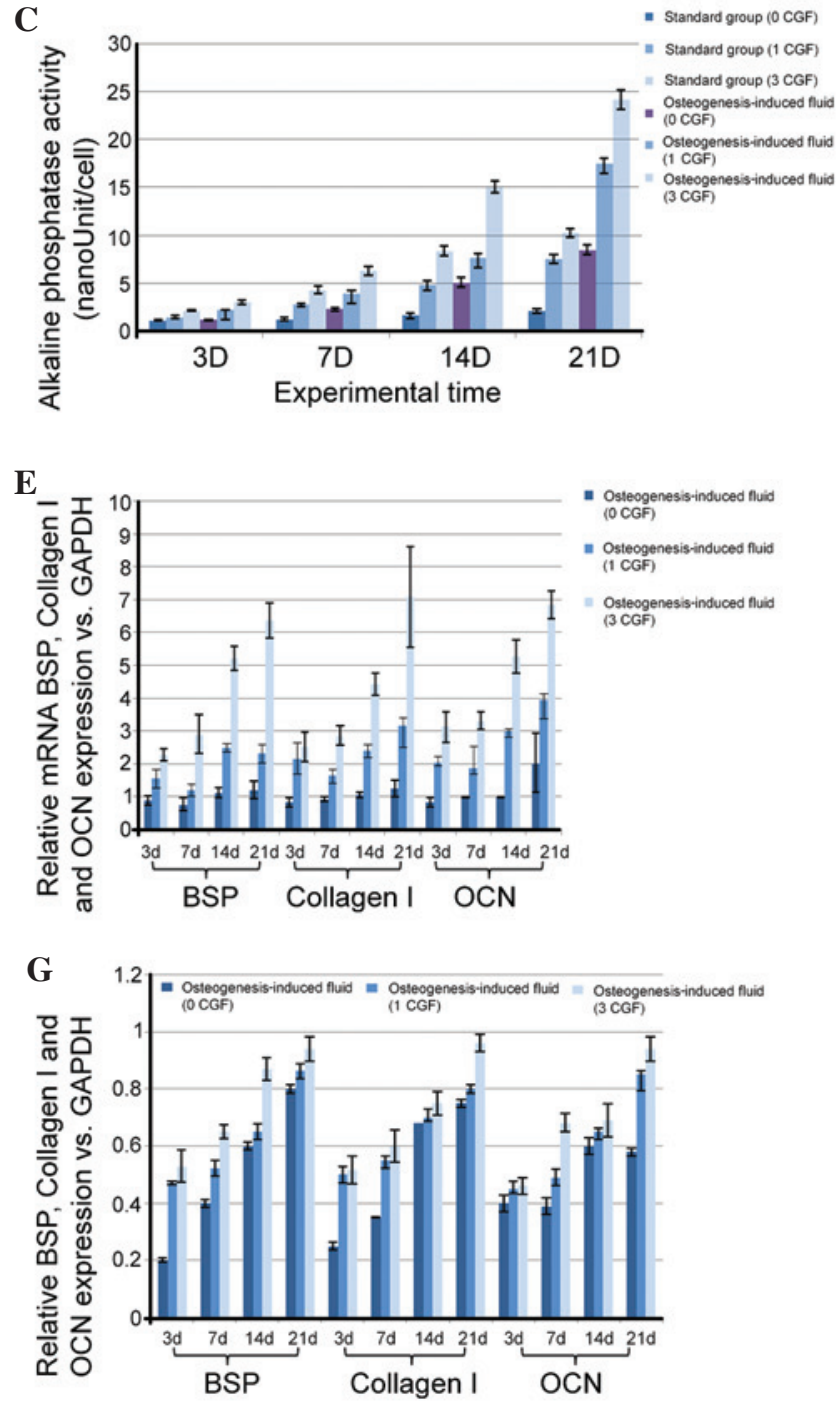

B

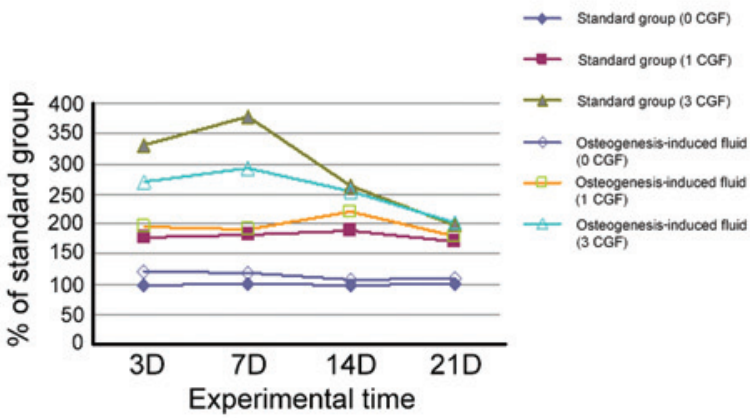

D

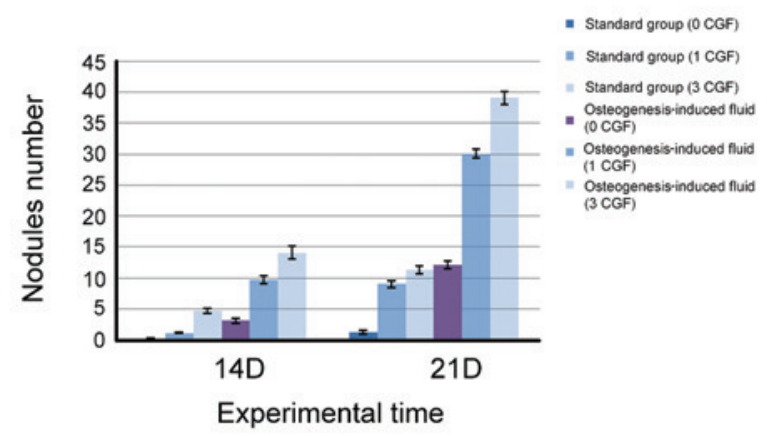

F

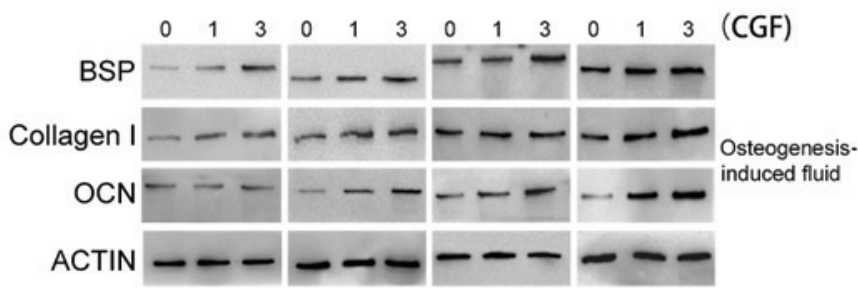

H

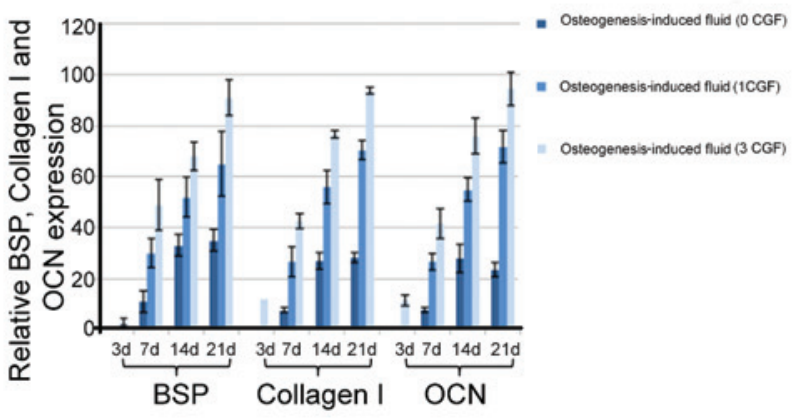

Figure 2. (A) Proliferation assessment of beagle PDLSCs in six different experimental conditions and at four experimental times. (B) MTT assay results of the test groups are expressed as mean percentages of the control. (C) Alkaline phosphatase activity quantification (nU/cell), and (D) the numeration of critical sized mineralization nodules in beagle PDLSCs cultures in six different experimental conditions. Relative expression of BSP, collagen I and OCN with osteogenesis-induced fluid based on (E) qPCR, (F and H) western blot analysis and (G) immunohistochemistry. Results are expressed as the mean \pm SD. PDLSCs, periodontal ligament stem cells; BSP, bone sialoprotein 2; OCN, osteocalcin.

time- and dose-dependent manner $(\mathrm{P}<0.05)$, and a significant increase appeared at 14 day. From the MTT assay, in standard culture conditions, the stimulation of proliferation ranged from 178 to $189 \%$ with 1 CGF and from 198 to $330 \%$ with 3 CGF (Fig. 2B). The stimulation level was stable with 1 CGF, but the cell activity peaked on day 7 with 3 CGF. The cultures with 3 CGF were more strongly stimulated compared with those with $1 \mathrm{CGF}(\mathrm{P}<0.05)$ at least up to 14 days. In the osteogenic medium, the stimulation of proliferation ranged between 178 and $196 \%$ with 1 CGF and between 201 and $292 \%$ with 3 CGF (Fig. 2B). The stimulation level was stable with 1 and 3 CGF. In addition, it was observed that the cultures with 3 CGF were more strongly stimulated than those with 1 CGF $(\mathrm{P}<0.05)$ throughout the experimental period. 


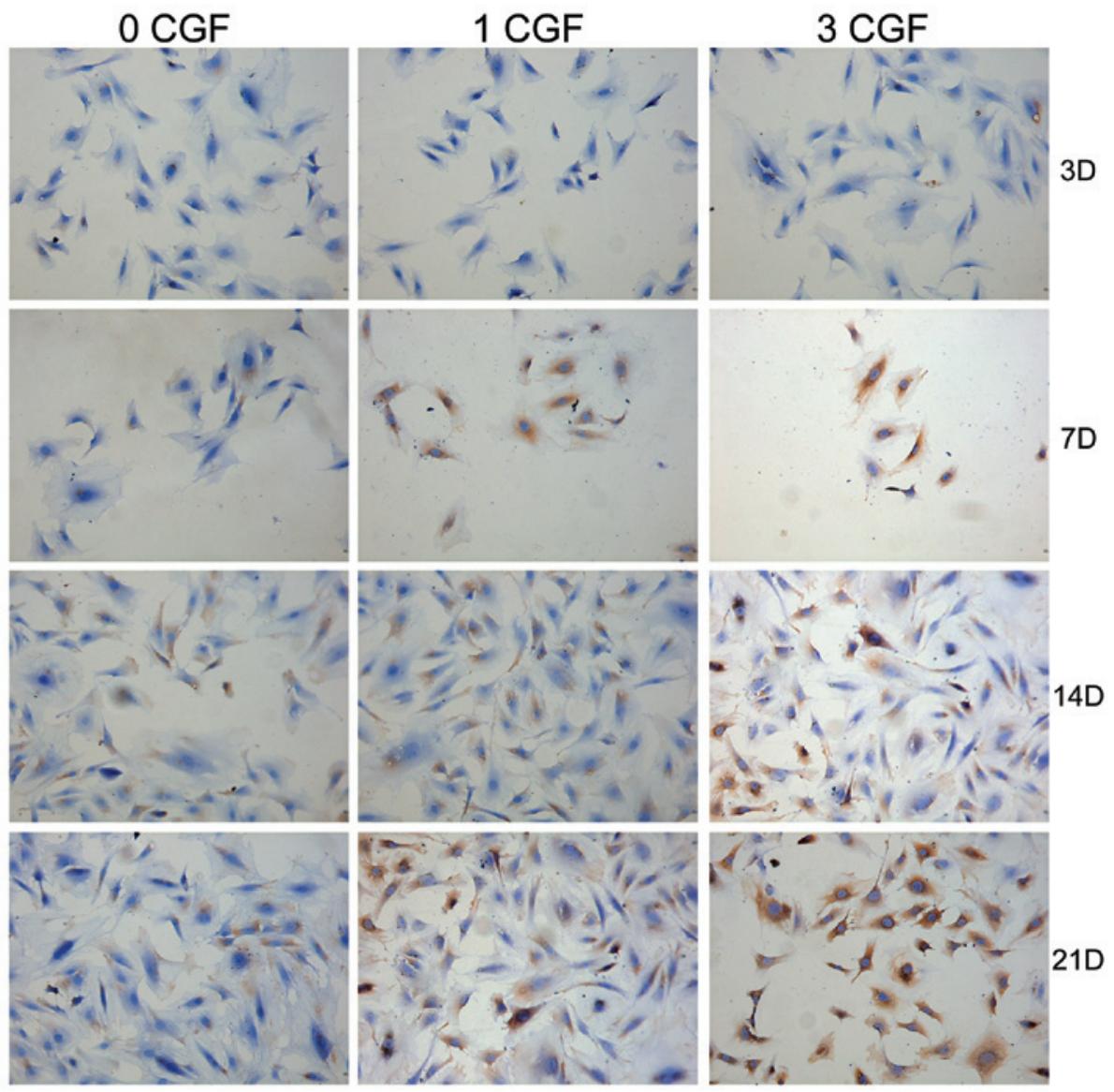

Figure 3. Relative expression of bone sialoprotein 2 BSP with osteogenesis-induced fluid observed by immunohistochemical analysis. DAB, chromogenic and hematoxylin staining was conducted. Bar, $100 \mu \mathrm{m}$. CGFs, concentrated growth factors.

Osteoinduction. ALP activity detection and mineralized nodule counts were performed to determine the effect of CGFs on the induction of osteogenic differentiation of PDLSCs cells. ALP activity was detected on days 3, 7, 14 and 21, and mineralized nodule counts were conducted on days 14 and 21 . In the presence of 1 or 3 CGFs, the ALP activities (Fig. 2C) and the number of mineralized nodules (Fig. 2D) were significantly higher $(\mathrm{P}<0.05)$ than the values of the respective control groups, and stimulation of differentiation was significantly higher $(\mathrm{P}<0.05)$ with $3 \mathrm{CGF}$ than with $1 \mathrm{CGF}$, regardless of the culture conditions.

The experiments performed showed that the CGFs exhibits a significant positive effect on PDLSC cell morphology due to osteogenic induction and differentiation. To the best of our knowledge, no previous study has investigated this event at the molecular level. In the present study, qPCR analysis was used to detect the expression of bone sialoprotein 2 (BSP), collagen I and osteocalcin (OCN) of different groups at each experimental check point during osteogenic induction. In Fig. 2E, the mRNA expression level of BSP showed an increasing trend along with the increasing quantity of CGF added $(\mathrm{P}<0.05)$. In addition, as the culture time increased, BSP mRNA expression was increased, however, it showed no significant increase for the first 7 days $(\mathrm{P}>0.05)$ and then significantly increased on day $14(\mathrm{P}<0.05)$. OCN and collagen I mRNA expression also showed the same increasing trend following CGF addition.
Furthermore, western blot analysis was used to analyze cell growth on the protein level. As shown in Fig. 2F, CGFs were observed to upregulate BSP protein expression in PDLSCs during the culture period $(\mathrm{P}<0.05)$. The quantitative measurement is shown in Fig. 2G. BSP protein expression in cells cultured in osteogenic medium was significantly increased with 3 CGF $(\mathrm{P}<0.05)$ on day 7 and 21 compared with 1 CGF. Collagen I protein expression levels also increased in a timeand dose-dependent manner, and peaked on day $14(\mathrm{P}<0.05)$. The OCN protein expression levels increased with time and peaked at day $21(\mathrm{P}<0.05)$.

Furthermore, immunohistochemical analysis of the flag factor BSP, collagen I and OCN protein expression was also performed during the osteogenic induction process at different experimental check points in all the experimental groups. As shown in Figs. 3-5, BSP, collagen I and OCN-positive expression, respectively, occurred at day 7 when co-cultured with CGF; however, the control group showed a positive expression at day 14. The results indicated that BSP, collagen I and OCN-positive expression increased (Fig. 2H) in a time- and dose-dependent manner $(\mathrm{P}<0.05)$.

\section{Discussion}

Adult stem cells are specific to adult tissues and organs. Under normal circumstances, the majority of the cells are in a resting state; however, damage to body tissues stimulates 


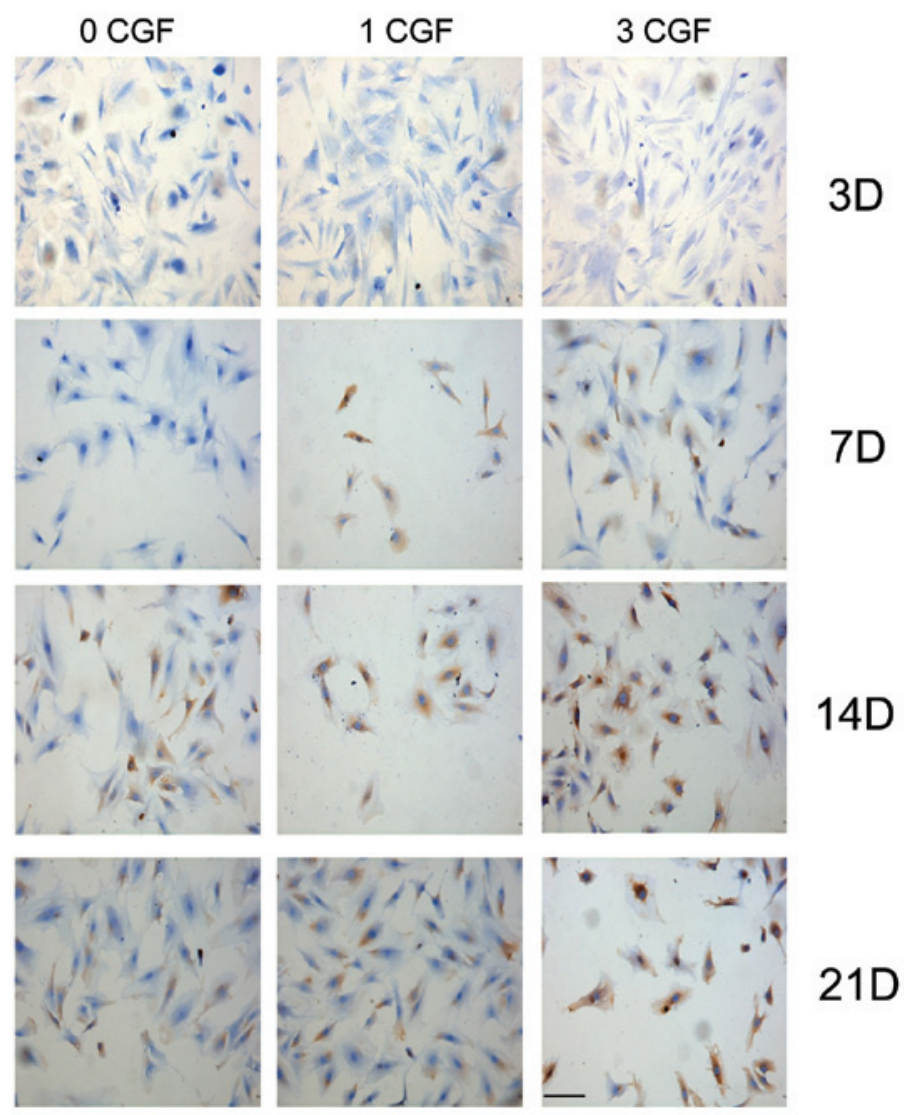

Figure 4. Relative expression of collgen I with osteogenesis-induced fluid observed by immunohistochemical analysis. DAB, chromogenic and hematoxylin staining was conducted. Bar, $100 \mu \mathrm{m}$. CGFs, concentrated growth factors.

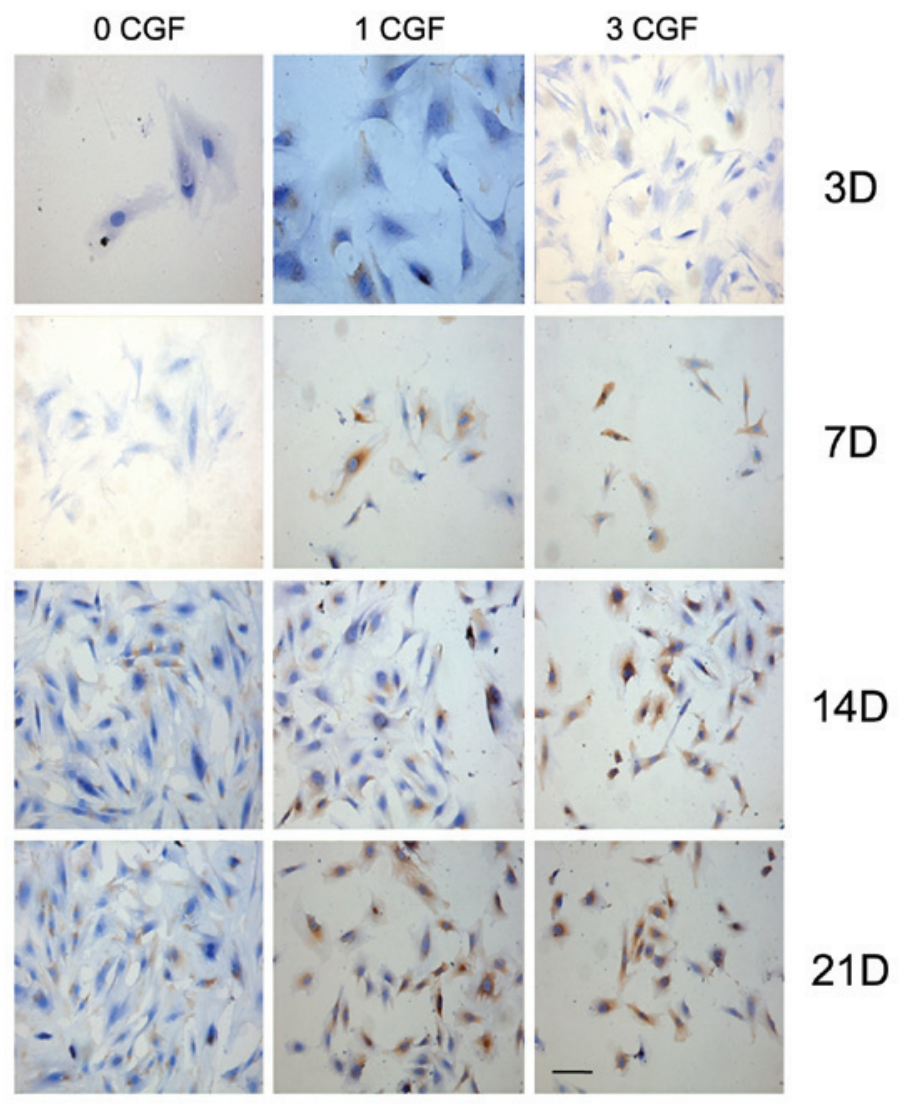

Figure 5. Relative expression of osteocalcin with osteogenesis-induced fluid observed by immunohistochemical analysis. DAB, chromogenic and hematoxylin staining was conducted. Bar, $100 \mu \mathrm{m}$. CGFs, concentrated growth factors. 
differentiation of the stem cells in the tissue. The stem cells differentiate into the damaged tissue cells and are involved in tissue repair and reconstruction. Therefore, in theory PDLSCs are the most direct and reliable seed cells for periodontal tissue regeneration. There is no ideal method for the isolation of adult stem cells. Generally, stem cells are separated and identified based on their colony-forming ability and specific biological and physical characteristics. As no specific markers have been identified for PDLSCs, they are predominanly identified by STRO-1, CD146 and other mesenchymal stem cell marker proteins and are isolated by the immunomagnetic bead separation method or flow cytometry. These methods are the same for bone marrow stem cells and dental pulp stem cells, which have similar organizational structure and embryonic origin to PDLSCs (21). As an increasing number of PDLSC studies have been conducted, STRO-1, CD146 and other mesenchymal stem cell surface markers and certain perivascular cell surface markers, such as CD105, CD106 and CD166, have been widely recognized. The STRO-1 antibody immunomagnetic or the flow cytometry separation methods are commonly known to be the most effective separation methods for PDLSCs $(4,20-23)$. In the present study, STRO- $1^{+}$cells were separated by flow cytometry and preliminary identification indicated that these cells possess high self-renewal capacity and multi-lineage differentiation potential.

Similar to PRF, the CGFs were only centrifuged once to avoid the loss of fibrinogen. The CGFs formed free modification gelatinous fiber blocks, which were convenient for operation and application. In the present study, CGFs were able to significantly stimulate PDLSC proliferation in a dose-dependent manner in vitro, in standard or differentiation medium. The mechanism responsible for the cell proliferation by CGFs may be explained as follows: CGFs are rich in a variety of growth factors, such as TGF- $\beta$, PDGF-AB, VEGF and IGF-I (24). These growth factors while functioning on their own (25-29), are also synergistic and create close contact tissue repair regulatory systems $(24,30,31)$. Unlike PRP, CGFs do not dissolve rapidly following application, instead, the strong fibrin gel in the matrix addition is slowly remodeled in a similar manner to a natural blood clot. Thus, CGFs prolonged the duration of growth factor action, which is conducive for the growth factor synergy (32-34), and enhances cell proliferation and osteogenic differentiation. Previous studies have demonstrated that the PRP may induce proliferation, but inhibits differentiation (16), and certain studies have indicated that high concentrations of PRP may also exhibit an inhibitory effect on the cultured cells (35). In the present study, this was not observed. To avoid any bias related to immune incompatibility, in the present study, CGF was obtained from the same donor as for the PDLSCs. CGFs exhibited no cytotoxic effect on the PDLSCs at the two doses tested similar to PRF (36). CGF also significantly promoted the proliferation of PDLSCs, and exhibited a dose-dependent effect on the activation and differentiation of the stem cells.

With the aid of the osteogenic inductive medium, PDLSCs develop osteogenically. The cells undergo multiple independent stages of differentiation in order to evolve into osteoblasts. The process includes: The conversion phrase, the proliferation phase, cell aggregation secretory phase and extracellular matrix calcification period (37). Throughout the differentiation process, the alkaline phosphatase activity, matrix mineralization, osteocalcin and collagen I are all specific markers for stem cell osteogenic differentiation. Osteoblasts synthesize different products in different stages. Collagen I is produced in the cell proliferation phase, cell aggregation secretory phase synthesizes alkaline phosphatase, and osteocalcin is produced in the extracellular matrix calcification phase (38). Thus, in the present study, the indicators that determine osteoblast differentiation of PDLSCs were mineralized nodule formation, alkaline phosphatase activity, type I collagen synthesis and specific protein expression, such as BSP and OCN. In the present study, ALP activity and mineralized nodule count were elevated by CGF in a time- and dose-dependent manner. qPCR, western blot analysis and immunohistochemical results were used to determine that CGF mediated the osteogenic differentiation of PDLSCs and the results show that CGF accelerates osteogenesis during the differentiation process. Similar the results have been demonstrated previously (39); thus, CGF may contribute to the differentiation of PDLSCs.

In conclusion, CGF significantly promotes the proliferation of PDLSCs and has a dose-dependent effect. In standard culture medium, CGF induces PDLSC osteogenic differentiation. In conditioned medium, CGFs significantly accelerate the osteogenesis transformation process of PDLSCs. CGF mediated culture medium either directly induces transformation or accelerates transformation in a dose-dependent manner. Future studies are required to identify the optimal induction dose of CGF, to determine the mechanism underlying the dose-dependent trend and to reproduce the results in in vivo studies. The present study has therefore provided an experimental basis for further PDLSCs clinical applications and studies.

\section{Acknowledgements}

This study was supported by a research grant (no.09411955100) from Tongji University to Professor Zuolin Wang, the 2010 Shanghai Committee of Science and Technology of China (grant no. 10XD1404500) and National Natural Science Foundation of China (grant no. 81271110).

\section{References}

1. Hench LL: Biomaterials: a forecast for the future. Biomaterials 19: 1419-1423, 1998.

2. Davo R, Malevez C and Rojas J: Immediate function in the atrophic maxilla using zygoma implants: a preliminary study. J Prosthet Dent 97 (Suppl 6): S44-S51, 2007.

3. Petrovic V and Stefanovic V: Dental tissue - new source for stem cells. ScientificWorldJournal 9: 1167-1177, 2009.

4. Gronthos S, Brahim J, Li W, et al: Stem cell properties of human dental pulp stem cells. J Dent Res 81: 531-535, 2002.

5. Seo BM, Miura M, Gronthos S, et al: Investigation of multipotent postnatal stem cells from human periodontal ligament. Lancet 364: 149-155, 2004.

6. Radtke S and Horn PA: Cells, niche, fate: meeting report on the 6th International Meeting of the Stem Cell Network North Rhine Westphalia. Cell Reprogram 13: 381-384, 2011.

7. Forte A, Galderisi U, Cipollaro M and Cascino A: Mesenchymal stem cells: a good candidate for restenosis therapy? Curr Vasc Pharmacol 7: 381-393, 2009.

8. Deryugina EI and Müller-Sieburg CE: Stromal cells in long-term cultures: keys to the elucidation of hematopoietic development? Crit Rev Immunol 13: 115-150, 1993.

9. Fini M, Giavaresi G, Torricelli P, et al: Osteoporosis and biomaterial osteointegration. Biomed Pharmacother 58: 487-493, 2004. 
10. Davies JE: Mechanisms of endosseous integration. Int J Prosthodont 11: 391-401, 1998.

11. Meyer U, Joos U, Mythili J, et al: Ultrastructural characterization of the implant/bone interface of immediately loaded dental implants. Biomaterials 25: 1959-1967, 2004.

12. Shields LB, Raque GH, Glassman SD, et al: Adverse effects associated with high-dose recombinant human bone morphogenetic protein-2 use in anterior cervical spine fusion. Spine (Phila Pa 1976) 31: 542-547, 2006.

13. Alanay A, Chen $C$, Lee $S$, et al: The adjunctive effect of a binding peptide on bone morphogenetic protein enhanced bone healing in a rodent model of spinal fusion. Spine (Phila Pa 1976) 33: 1709-1713, 2008.

14. Glassman SD, Carreon LY, Campbell MJ, et al: The perioperative cost of Infuse bone graft in posterolateral lumbar spine fusion. Spine J 8: 443-448, 2008.

15. Assoian RK, Grotendorst GR, Miller DM and Sporn MB: Cellular transformation by coordinated action of three peptide growth factors from human platelets. Nature 309: 804-806, 1984.

16. Vogel JP, Szalay K, Geiger F, Kramer M, Richter W and Kasten P: Platelet-rich plasma improves expansion of human mesenchymal stem cells and retains differentiation capacity and in vivo bone formation in calcium phosphate ceramics. Platelets 17: 462-469, 2006.

17. Choukroun J, Adda F, Schoeffler C and Vervelle A: Une opportunité en paro-implantologie: le PRF. Implantodontie 42: 55-62, 2001.

18. Corigliano M, Sacco L and Baldoni E: CGF- una proposta terapeutica per la medicina rigenerativa. Odontoiatria - nol anno XXIX - Maggio 1: 69-81, 2010.

19. Sohn DS, Heo JU, Kwak DH, et al: Bone regeneration in the maxillary sinus using an autologous fibrin-rich block with concentrated growth factors alone. Implant Dent 20: 389-395, 2011.

20. He H, Yu J, Cao J, et al: Biocompatibility and osteogenic capacity of periodontal ligament stem cells on nHAC/PLA and HA/TCP scaffolds. J Biomater Sci Polym Ed: Jun 16, 2010 (Epub ahead of print).

21. Mrozik K, Gronthos S, Shi S and Bartold PM: A method to isolate, purify, and characterize human periodontal ligament stem cells. Methods Mol Biol 666: 269-284, 2010.

22. Coura GS, Garcez RC, de Aguiar CB, Alvarez-Silva M, Magini RS and Trentin AG: Human periodontal ligament: a niche of neural crest stem cells. J Periodontal Res 43: 531-536, 2008.

23. Lee UL, Jeon SH, Park JY and Choung PH: Effect of platelet-rich plasma on dental stem cells derived from human impacted third molars. Regen Med 6: 67-79, 2011.

24. Rodella LF, Favero G, Boninsegna R, et al: Growth factors, CD34 positive cells, and fibrin network analysis in concentrated growth factors fraction. Microsc Res Tech 74: 772-777, 2011.

25. Zhao L, Jiang S and Hantash BM: Transforming growth factor betal induces osteogenic differentiation of murine bone marrow stromal cells. Tissue Eng Part A 16: 725-733, 2010.
26. Zeiter S, Lezuo P and Ito K: Effect of TGF beta1, BMP-2 and hydraulic pressure on chondrogenic differentiation of bovine bone marrow mesenchymal stromal cells. Biorheology 46: 45-55, 2009.

27. Wirz S, Dietrich M, Flanagan TC, et al: Influence of plateletderived growth factor-AB on tissue development in autologous platelet-rich plasma gels. Tissue Eng Part A 17: 1891-1899, 2011.

28. Nakano N, Nakai Y, Seo TB, et al: Characterization of conditioned medium of cultured bone marrow stromal cells. Neurosci Lett 483: 57-61, 2010.

29. Fang Y, Wang LP, Du FL, Liu WJ and Ren GL: Effects of insulin-like growth factor I on alveolar bone remodeling in diabetic rats. J Periodontal Res 48: 144-150, 2013.

30. Sunitha Raja V and Munirathnam Naidu E: Platelet-rich fibrin: evolution of a second-generation platelet concentrate. Indian J Dent Res 19: 42-46, 2008.

31. Dohan DM, Choukroun J, Diss A, et al: Platelet-rich fibrin (PRF): a second-generation platelet concentrate. Part II: platelet-related biologic features. Oral Surg Oral Med Oral Pathol Oral Radiol Endod 101: e45-e50, 2006.

32. Kang YH, Jeon SH, Park JY, et al: Platelet-rich fibrin is a Bioscaffold and reservoir of growth factors for tissue regeneration. Tissue Eng Part A 17: 349-359, 2011.

33. Dohan Ehrenfest DM, de Peppo GM, Doglioli P and Sammartino G: Slow release of growth factors and thrombospondin-1 in Choukroun's platelet-rich fibrin (PRF): a gold standard to achieve for all surgical platelet concentrates technologies. Growth Factors 27: 63-69, 2009.

34. Dohan Ehrenfest DM, Rasmusson L and Albrektsson T: Classification of platelet concentrates: from pure platelet-rich plasma (P-PRP) to leucocyte- and platelet-rich fibrin (L-PRF). Trends Biotechnol 27: 158-167, 2009.

35. Graziani F, Ivanovski S, Cei S, Ducci F, Tonetti M and Gabriele M: The in vitro effect of different PRP concentrations on osteoblasts and fibroblasts. Clin Oral Implants Res 17: 212-219, 2006.

36. Dohan Ehrenfest DM, Doglioli P, de Peppo GM, Del Corso M and Charrier JB: Choukroun's platelet-rich fibrin (PRF) stimulates in vitro proliferation and differentiation of human oral bone mesenchymal stem cell in a dose-dependent way. Arch Oral Biol 55: 185-194, 2010.

37. Yoshikawa T, Nakajima H, Takakura $\mathrm{Y}$ and Nonomura A: Osteogenesis with cryopreserved marrow mesenchymal cells. Tissue Eng 11: 152-160, 2005.

38. Liu G, Shu C, Cui L, Liu W and Cao Y: Tissue-engineered bone formation with cryopreserved human bone marrow mesenchymal stem cells. Cryobiology 56: 209-215, 2008.

39. Huang FM, Yang SF, Zhao JH and Chang YC: Platelet-rich fibrin increases proliferation and differentiation of human dental pulp cells. J Endod 36: 1628-1632, 2010. 